\title{
Class number calculation using Siegel functions
}

\author{
T. Fukuda and K. Komatsu
}

\begin{abstract}
We propose a fast method of calculating the $p$-part of the class numbers in certain non-cyclotomic $\mathbb{Z}_{p}$-extensions of an imaginary quadratic field using elliptic units constructed by Siegel functions. We carried out practical calculations for $p=3$ and determined $\lambda$-invariants of such $\mathbb{Z}_{3}$-extensions which were not known in our previous paper.
\end{abstract}

\section{Introduction}

Let $K$ be an imaginary quadratic field and $p$ an odd prime number which splits into two distinct primes $\mathfrak{p}$ and $\overline{\mathfrak{p}}$ in $K$. We denote by $K_{n}^{\prime}=K\left(\mathfrak{p}^{n+1}\right)$ the ray class field of $K$ modulo $\mathfrak{p}^{n+1}$ and $K_{\infty}^{\prime}=\bigcup_{n=0}^{\infty} K_{n}^{\prime}$. Then there exists a unique $\mathbb{Z}_{p}$-extension $K_{\infty}$ of $K$ in $K_{\infty}^{\prime}$. We denote by $K_{n}$ the $n$th layer of $K_{\infty}$ over $K$.

In a previous paper [3] we studied the Iwasawa invariant $\lambda\left(K_{\infty} / K\right)$ for $p=3$, while $\mu\left(K_{\infty} / K\right)$ is known to be zero by $[4,7]$. Our investigation was based on the calculation in $K_{2}$. We were not able to handle $K_{n}(n \geqslant 3)$ for lack of a fine algorithm. In the present paper we develop a new algorithm based on the structure of the group of elliptic units and calculate the 3 -part of the class number $h\left(K_{n}\right)$ of $K_{n}(1 \leqslant n \leqslant 5)$. We are now able to consider $\lambda\left(K_{\infty} / K\right)$ by observing directly the growth of the 3 -part of $h\left(K_{n}\right)$.

We illustrate, for an odd prime number $p$, how to calculate the $p$-part of $h\left(K_{n}\right)$. As usual, for a Galois extension $L / F$ of algebraic number fields, we denote by $G(L / F)$ the Galois group of $L$ over $F$ and by $N_{L / F}$ the norm mapping of $L$ over $F$. We begin by explaining how to construct $K_{n}$ explicitly. We assume that $K$ is different from both $\mathbb{Q}(\sqrt{-1})$ and $\mathbb{Q}(\sqrt{-3})$. As in [3], we are interested in $K_{\infty} / K$ in which $\mathfrak{p}$ is totally ramified. Therefore $\widetilde{K} \cap K_{\infty}=K$, where $\widetilde{K}$ means the Hilbert class field of $K$. Let $a_{1}, a_{2}$ be rational numbers and $\tau$ a complex number with positive imaginary part. Then the Siegel function is defined to be

$$
g\left(a_{1}, a_{2}\right)(\tau)=-q_{\tau}^{(1 / 2)\left(a_{1}^{2}-a_{1}+1 / 6\right)} e^{2 \pi i a_{2}\left(a_{1}-1\right) / 2}\left(1-q_{z}\right) \prod_{n=1}^{\infty}\left(1-q_{\tau}^{n} q_{z}\right)\left(1-q_{\tau}^{n} q_{z}^{-1}\right),
$$

where $q_{\tau}=e^{2 \pi i \tau}, q_{z}=e^{2 \pi i z}, z=a_{1} \tau+a_{2}$ and $i=\sqrt{-1}$. Then $g\left(a_{1}, a_{2}\right)(\tau)$ is a modular function of some level and $K_{n}$ is generated by special values of $g\left(a_{1}, a_{2}\right)$. We refer [5, Chapter 2] for the various properties of the Siegel function.

Let $\omega_{1}$ and $\omega_{2}$ be elements of $K$ with imaginary part $\operatorname{Im}\left(\omega_{1} / \omega_{2}\right)>0$ such that $\mathfrak{p}^{n+1}=$ $\mathbb{Z} \omega_{1}+\mathbb{Z} \omega_{2}$. Since $(p)=\mathfrak{p p}^{\prime}$, there exist integers $r, s \in \mathbb{Z}$ with

$$
\frac{r}{p^{n+1}} \omega_{1}+\frac{s}{p^{n+1}} \omega_{2}=1 \text {. }
$$

Then $g\left(r / p^{n+1}, s / p^{n+1}\right)\left(\omega_{1} / \omega_{2}\right)^{12 p^{n+1}}$ is in $K_{n}^{\prime}$ by [5, p. 234, Theorem 1.1]. We put

$$
f(\tau)=\left(g\left(\frac{r}{p^{n+1}}, \frac{s}{p^{n+1}}\right)(\tau) / g\left(\frac{r(1+p)}{p^{n+1}}, \frac{s(1+p)}{p^{n+1}}\right)(\tau)\right)^{4} .
$$

Received 27 February 2014; revised 23 May 2014.

2010 Mathematics Subject Classification 11R29, 11R23 (primary).

Contributed to the Algorithmic Number Theory Symposium XI, GyeongJu, Korea, 6-11 August 2014. 
We know that $f(\tau)$ is independent of $r, s$ by [5, p. 33, Proposition 1.3]. Then there exists a unique $3 p^{n+1}$ th root of unity $\zeta$ such that $f\left(\omega_{1} / \omega_{2}\right) \zeta \in K_{n}^{\prime}$ by [3, p. 472]. We put

$$
\eta_{n}=N_{K_{n}^{\prime} / K_{n}}\left(f\left(\frac{\omega_{1}}{\omega_{2}}\right) \zeta\right) .
$$

Let $\Gamma$ be the Galois group $G\left(K_{\infty} / K\right)$ and $\gamma$ is topological generator of $\Gamma$. We put

$$
\mathcal{E}_{n}=\left\langle\eta_{n}, \eta_{n}^{\gamma}, \ldots, \eta_{n}^{\gamma^{p^{n}-2}}\right\rangle .
$$

Let $E_{n}$ be the unit group of $K_{n}$. Then it is well known that the group index $\left(E_{n}: \mathcal{E}_{n}\right)$ is finite $\left[\boldsymbol{5}\right.$, p. 323 , Theorem 4.1]. We note that $\eta_{n}, \eta_{n}^{\gamma}, \ldots, \eta_{n}^{\gamma^{p^{n}-2}}$ form a free basis of $\mathcal{E}_{n}$. Let $E_{n}^{\prime}$ be the subgroup of $E_{n}$ such that $E_{n}^{\prime} / \mathcal{E}_{n}$ is the $p$-Sylow subgroup of $E_{n} / \mathcal{E}_{n}$. Let $p^{e_{n}}$ be the exact power of $p$ dividing the class number $h\left(K_{n}\right)$ of $K_{n}$. Then we have

$$
p^{e_{n}}=p^{e_{0}}\left(E_{n}^{\prime}: \mathcal{E}_{n}\right)
$$

by [5, p. 323, Theorem 4.1]. Our main purpose of this paper is to prove the following theorem.

TheOREM 1.1. Let the notation and assumptions be as above. If $e_{n}-e_{n-1}=1$ for some integer $n \geqslant 1$, then we have $e_{n+1}-e_{n} \leqslant 1$.

Owing to [2, Theorem 1], we may convert Theorem 1.1 into the following version.

Corollary 1.2. If $e_{n}-e_{n-1} \leqslant 1$ for some integer $n \geqslant 1$, then we have $e_{m}-e_{m-1} \leqslant 1$ for all integers $m \geqslant n$.

As an application of Corollary 1.2, we show an efficient algorithm for calculating $e_{n}$ in the case $e_{1}-e_{0}=1$ in $\S 3$.

\section{Proof of theorem}

Preparatory to proving Theorem 1.1, we summarize as lemmas properties of $E_{n}, E_{n}^{\prime}$ and $\mathcal{E}_{n}$ which were defined in the previous section.

Lemma 2.1. We have $\mathcal{E}_{n} \cap E_{n-1}=\mathcal{E}_{n-1}$ for $n \geqslant 1$.

Proof. We write $s=p^{n-1}-1$ and $r=p^{n}-p^{n-1}-1$. Put

$$
\eta=\eta_{n}^{x_{0}+x_{1} \gamma+\ldots+x_{p^{n}-2} \gamma^{p^{n}-2}}
$$

with rational integers $x_{i}$. We assume $\eta \in E_{n-1}$. Then $\eta^{\gamma^{p^{n-1}}}=\eta$, which implies

$$
\eta^{\gamma^{p^{n-1}}}=\eta_{n}^{\sum_{i=0}^{p^{n}-2}\left(x_{i}-x_{r}\right) \gamma^{i+p^{n-1}}}=\eta
$$

by $N_{K_{n} / K}\left(\eta_{n}\right)=1$. Hence we have

$$
\begin{aligned}
x_{i}-x_{r} & =x_{i+p^{n-1}} \quad(0 \leqslant i \leqslant r-1), \\
x_{i}-x_{r} & =x_{i+p^{n-1}-p^{n}} \quad\left(r+1 \leqslant i \leqslant p^{n}-2\right), \\
-x_{r} & =x_{p^{n-1}-1},
\end{aligned}
$$

which means $x_{0}-p x_{r}=x_{0}$. This shows $x_{r}=0$ and $x_{p^{n-1}-1}=0$. It is known that $N_{K_{n} / K_{n-1}}\left(\eta_{n}\right)=\eta_{n-1}$ by [5, Theorem 1.3, p. 237]. Hence, noting the uniqueness of $\zeta$, we have

$$
\eta=N_{K_{n} / K_{n-1}}\left(\eta_{n}^{x_{0}+x_{1} \gamma+\ldots+x_{s-1} \gamma^{s-1}}\right)=\eta_{n-1}^{x_{0}+x_{1} \gamma+\ldots+x_{s-1} \gamma^{s-1}} .
$$


Lemma 2.2. We have $p^{e_{n}-e_{n-1}}=\left(E_{n}^{\prime}: \mathcal{E}_{n} E_{n-1}^{\prime}\right)$ for $n \geqslant 1$.

Proof. Since $\mathcal{E}_{n} \cap E_{n-1}^{\prime}=\mathcal{E}_{n-1}$ by Lemma 2.1 , we have $\left(\mathcal{E}_{n} E_{n-1}^{\prime}: \mathcal{E}_{n}\right)=\left(E_{n-1}^{\prime}: \mathcal{E}_{n-1}\right)$. Hence we have

$$
\begin{aligned}
p^{e_{n}}=\left(E_{n}^{\prime}: \mathcal{E}_{n}\right) & =\left(E_{n}^{\prime}: \mathcal{E}_{n} E_{n-1}^{\prime}\right)\left(\mathcal{E}_{n} E_{n-1}^{\prime}: \mathcal{E}_{n}\right) \\
& =\left(E_{n}^{\prime}: \mathcal{E}_{n} E_{n-1}^{\prime}\right)\left(E_{n-1}^{\prime}: \mathcal{E}_{n-1}\right) \\
& =p^{e_{n-1}}\left(E_{n}^{\prime}: \mathcal{E}_{n} E_{n-1}^{\prime}\right) .
\end{aligned}
$$

Lemma 2.3. If $E_{n}^{\prime} / \mathcal{E}_{n}$ is non-trivial, then there exists an element $\eta$ in $\mathcal{E}_{n}$ with $\eta \notin \mathcal{E}_{n}^{p}$ and $\eta^{\gamma-1} \in \mathcal{E}_{n}^{p}$, where $\mathcal{E}_{n}^{p}=\left\{\varepsilon^{p} \mid \varepsilon \in \mathcal{E}_{n}\right\}$.

Proof. Since $E_{n}^{\prime} / \mathcal{E}_{n}$ is a non-trivial $p$-group, there exists an element $u$ in $E_{n}^{\prime}$ such that $u \notin \mathcal{E}_{n}$, $u^{\gamma-1} \in \mathcal{E}_{n}$ and $u^{p} \in \mathcal{E}_{n}$. We put $\eta=u^{p}$. Then $\eta \notin \mathcal{E}_{n}^{p}$ and $\eta^{\gamma-1}=\left(u^{\gamma-1}\right)^{p} \in \mathcal{E}_{n}^{p}$ because $E_{n}$ does not contain a non-trivial $p$ th root of unity.

Let $H$ be a subgroup of $E_{n}$ and $u, v$ elements of $E_{n}$. From now on, we write $u \equiv v(\bmod H)$ if $u v^{-1} \in H$. We put $T=\gamma-1$ as usual.

Lemma 2.4. There exists an element $f_{n}(T)$ in $\mathbb{Z}[T]$ which satisfies

$$
\eta_{n}^{T^{p^{n}-p^{n-1}}}=\eta_{n-1} \eta_{n}^{-p\left(1+T f_{n}(T)\right)} .
$$

Proof. Since $\sum_{i=0}^{p-1}(T+1)^{i p^{n-1}} \equiv \sum_{i=0}^{n-1}\left(T^{p^{n-1}}+1\right)^{i} \equiv T^{p^{n}-p^{n-1}}(\bmod p)$, we see that $\sum_{i=0}^{n-1}(T+1)^{i p^{n-1}}-T^{p^{n}-p^{n-1}} \in p \mathbb{Z}[T]$. Hence

$$
f_{n}(T)=\frac{\sum_{i=0}^{p-1}(T+1)^{i p^{n-1}}-T^{p^{n}-p^{n-1}}-p}{p T}
$$

is contained in $\mathbb{Z}[T]$ and

$$
\eta_{n-1}=N_{K_{n} / K_{n-1}}\left(\eta_{n}\right)=\eta_{n}^{\sum_{i=0}^{p-1}(T+1)^{i p^{n-1}}}=\eta_{n}^{T^{p^{n}-p^{n-1}}+p+p T f_{n}(T)}
$$

from which we derive the desired equality.

Proof of Theorem 1. We assume $e_{n}-e_{n-1}=1$ and $e_{n+1}-e_{n} \geqslant 2$ and derive a contradiction. We write $r=p^{n}-p^{n-1}-1$. Let $\left\{\xi_{1}, \xi_{2}, \ldots, \xi_{p^{n-1}-1}\right\}$ be a free basis of $E_{n-1}^{\prime}$ and put

$$
V_{n}=\left\langle\xi_{1}, \xi_{2}, \ldots, \xi_{p^{n-1}-1}, \eta_{n}, \eta_{n}^{\gamma}, \ldots, \eta_{n}^{\gamma^{r}}\right\rangle
$$

Since $\left\{\eta_{n}, \eta_{n}^{\gamma}, \ldots, \eta_{n}^{\gamma^{p^{n}-2}}\right\}$ is a free basis of $\mathcal{E}_{n}$ and since $N_{K_{n} / K_{n-1}}\left(\eta_{n}\right)=\eta_{n-1}$, we have $V_{n}=E_{n-1}^{\prime} \mathcal{E}_{n}$. We note

$$
V_{n}=\left\langle\xi_{1}, \xi_{2}, \ldots, \xi_{p^{n-1}-1}, \eta_{n}, \eta_{n}^{T}, \ldots, \eta_{n}^{T^{r}}\right\rangle
$$

Since $e_{n}-e_{n-1}=1$, there exist $v_{n} \in V_{n}, \varepsilon \in E_{n}^{\prime}-V_{n}$ and $x_{i}, y_{i} \in\{0,1, \ldots, p-1\}$ such that

$$
\varepsilon^{p}=v_{n}=\xi_{1}^{x_{1}} \xi_{2}^{x_{2}} \ldots \xi_{p^{n-1}-1}^{x_{p^{n-1}}} \eta_{n}^{y_{0}+y_{1} T+\ldots+y_{r} T^{r}}
$$

and $v_{n}^{T} \equiv 1\left(\bmod V_{n}^{p}\right)$ by Lemma 2.3. Since $\eta_{n}^{T^{r}} \equiv \eta_{n-1}\left(\bmod V_{n}^{p}\right)$ by Lemma 2.4, 
we have

$$
\begin{aligned}
v_{n}^{T} & \equiv \xi_{1}^{x_{1} T} \ldots \xi_{p^{n-1}-1}^{x_{p^{n-1}} T} \eta_{n-1}^{y_{r}} \eta_{n}^{-y_{r} p\left(1+T f_{n}(T)\right)} \eta_{n}^{y_{0} T+y_{1} T^{2}+\ldots+y_{r-1} T^{r}} \\
& \equiv \xi_{1}^{x_{1} T} \ldots \xi_{p^{p^{n-1}-1}}^{x_{n-1} T} \eta_{n-1}^{y_{r}} \eta_{n}^{y_{0} T+y_{1} T^{2}+\ldots+y_{r-1} T^{r}} \quad\left(\bmod V_{n}^{p}\right)
\end{aligned}
$$

Hence we have $y_{0}=y_{1}=\ldots=y_{r-1}=0$ and $y_{r} \neq 0$ by $e_{n}-e_{n-1}=1$. We may assume $y_{r}=1$. Hence there exists an element $\xi \in E_{n-1}^{\prime}$ with

$$
v_{n}=\xi \eta_{n}^{(\gamma-1)^{r}}
$$

such that $v_{n}^{1 / p} \in E_{n}^{\prime}$. This means $E_{n}^{\prime}=\left\langle V_{n} \cup\left\{v_{n}^{1 / p}\right\}\right\rangle$ by $e_{n}-e_{n-1}=1$. Since $\left(v_{n}^{1 / p}\right)^{T}=$ $\left(\xi^{T} \eta_{n-1} \eta_{n}^{-p\left(1+T f_{n}(T)\right)}\right)^{1 / p}$, there exists an element $\xi^{\prime} \in E_{n-1}^{\prime}$ with

$$
\left(v_{n}^{1 / p}\right)^{T}=\xi^{\prime} \eta_{n}^{-\left(1+T f_{n}(T)\right)} .
$$

We put

$$
V_{n+1}=\left\langle E_{n}^{\prime} \cup\left\{\eta_{n+1}, \eta_{n+1}^{T}, \ldots, \eta_{n+1}^{T^{r^{\prime}}}\right\}\right\rangle,
$$

where $r^{\prime}=p^{n+1}-p^{n}-1$. Then there exist $v_{n+1} \in V_{n+1}, \xi^{*} \in E_{n-1}^{\prime}, a_{i}, b_{i} \in\{0,1, \ldots, p-1\}$ and $\varepsilon^{*} \in E_{n+1}^{\prime}-V_{n+1}$ such that

$$
\varepsilon^{* p}=v_{n+1}=\xi^{*} \eta_{n}^{a_{0}+a_{1} T+\ldots+a_{r-1} T^{r-1}}\left(v_{n}^{1 / p}\right)^{a_{r}} \eta_{n+1}^{b_{0}+b_{1} T+\ldots+b_{r^{\prime}} T^{r^{\prime}}}
$$

and $v_{n+1}^{T} \equiv 1\left(\bmod V_{n+1}^{p}\right)$ by Lemma 2.3 and the assumption $e_{n+1}-e_{n} \geqslant 2$. Since $\eta_{n+1}^{T^{r^{\prime}+1}} \equiv \eta_{n}$ $\left(\bmod V_{n+1}^{p}\right)$ by Lemma 2.4 , we have

$$
\begin{aligned}
& v_{n+1}^{T} \equiv\left(\xi^{*}\right)^{T} \eta_{n}^{a_{o} T+\ldots+a_{r-2} T^{r-2}}\left(v_{n} \xi^{-1}\right)^{a_{r-1}} \\
& \cdot\left(\xi^{*} \eta_{n}^{-\left(1+T f_{n}(T)\right)}\right)^{a_{r}} \eta_{n+1}^{b_{0} T+\ldots+b_{r^{\prime}-1} T^{r^{\prime}-1}} \eta_{n}^{b_{r^{\prime}}} \\
& \equiv 1 \quad\left(\bmod V_{n+1}^{p}\right) \text {. }
\end{aligned}
$$

This shows $b_{0}=b_{1}=\ldots=b_{r-1}=0$ and $a_{r}=b_{r^{\prime}} \neq 0$. Since $b_{r^{\prime}}$ is prime to $p$, we may assume $a_{r}=1$. Hence there exists an element $\xi^{\prime \prime} \in V_{n}$ with

$$
v_{n+1}=\xi^{\prime \prime} v_{n}^{1 / p} \eta_{n+1}^{T^{r^{\prime}}}
$$

Moreover, we have $\left(v_{n+1}^{1 / p}\right)^{T}=\xi^{\prime \prime \prime} \eta_{n+1}^{-\left(1+T f_{n+1}(T)\right)}$ for some $\xi^{\prime \prime \prime} \in E_{n}^{\prime}$ by $\eta_{n+1}^{T^{r^{\prime}+1}}=\eta_{n}$ $\eta_{n+1}^{-p\left(1+T f_{n+1}(T)\right)}$. We put $V_{n+1}^{\prime}=\left\langle V_{n+1} \cup\left\{v_{n+1}^{1 / p}\right\}\right\rangle$. Then there exist $\varepsilon^{\prime} \in E_{n+1}^{\prime}, v_{n+1}^{\prime} \in V_{n+1}$, $\eta^{*} \in V_{n}$ and $y, z_{0}, \ldots, z_{r^{\prime}} \in\{0,1, \ldots, p-1\}$ with

$$
\varepsilon^{\prime p}=v_{n+1}^{\prime}=\eta^{*}\left(v_{n}^{1 / p}\right)^{y} \eta_{n+1}^{z_{0}+z_{1} T+\ldots+z_{r^{\prime}-1} T^{r^{\prime}-1}}\left(v_{n+1}^{1 / p}\right)^{z_{r^{\prime}}}
$$

and $\left(v_{n+1}^{\prime}\right)^{T} \equiv 1\left(\bmod V_{n+1}^{\prime p}\right)$ by the assumption $e_{n+1}-e_{n} \geqslant 2$. Since

$$
\begin{aligned}
\left(v_{n+1}^{\prime}\right)^{T} \equiv & \left(\eta^{*}\right)^{T}\left(\xi^{\prime} \eta_{n}^{-\left(1+T f_{n}(T)\right)}\right)^{y} \eta_{n+1}^{z_{0} T+\ldots+z_{r^{\prime}-1} T^{r^{\prime}-1}} \\
& \left.\cdot\left(\xi^{\prime \prime-1} v_{n}^{-1 / p}\right)^{z_{r^{\prime}-1}\left(\xi^{\prime \prime \prime}\right.} \eta_{n+1}^{-\left(1+f_{n+1}(T)\right)}\right)^{z_{r^{\prime}}} \\
\equiv & 1 \quad\left(\bmod V_{n+1}^{\prime p}\right),
\end{aligned}
$$

we have $z_{0}=z_{1}=\ldots=z_{r^{\prime}}=0$. This contradicts the assumptions. 


\section{Algorithm for constructing $E_{n}^{\prime}$}

As we explain in the later section, we often meet the situation $e_{1}-e_{0}=1$. In this case, we are able to develop an efficient algorithm for constructing $E_{n}^{\prime}$. By Corollary 1.2 and $(2.2), E_{n}^{\prime} / \mathcal{E}_{n}$ is a cyclic group with order $\left|E_{n}^{\prime} / \mathcal{E}_{n}\right| \leqslant p^{n}$. We assume $\left|E_{n}^{\prime} / \mathcal{E}_{n}\right|=p^{n}$ and construct $E_{n}^{\prime}$ as follows.

Based on the cyclicity of $E_{n}^{\prime} / \mathcal{E}_{n}$, there exist unique subgroups $V_{n, k}(0 \leqslant k \leqslant n)$ which satisfy

$$
\begin{aligned}
& \mathcal{E}_{n}=V_{n, 0} \subset V_{n, 1} \subset V_{n, 2} \subset \ldots \subset V_{n, n}=E_{n}^{\prime}, \\
& \left(V_{n, k+1}: V_{n, k}\right)=p
\end{aligned}
$$

We write $V_{k}$ for $V_{n, k}$.

Let $r=p^{n}-2$ and $\varepsilon_{i}=\eta_{n}^{\gamma^{i}}(0 \leqslant i \leqslant r)$. Then $V_{k}$ has the form

$$
V_{k}=\left\langle\varepsilon_{0}, \varepsilon_{1}, \ldots, \varepsilon_{r-1}, v_{k}\right\rangle
$$

with $v_{k} \in E_{n}^{\prime}$. Note that $v_{0}=\varepsilon_{r}$. We explain how to construct $v_{k+1}$ from $v_{k}$. By an argument similar to the proof of Lemma 2.3, we may assume $v_{k+1}^{p} \in V_{k}$ and $v_{k+1}^{p(1-\gamma)} \in V_{k}^{p}$. Namely, we search for integers $x_{i k}, y_{i k}$ and $v_{k+1} \in E_{n}^{\prime}$ satisfying

$$
\begin{aligned}
v_{k+1}^{p} & =\left(\prod_{i=0}^{r-1} \varepsilon_{i}^{x_{i k}}\right) v_{k}, \\
v_{k+1}^{p(1-\gamma)} & =\left(\left(\prod_{i=0}^{r-1} \varepsilon_{i}^{y_{i k}}\right) v_{k}^{y_{r k}}\right)^{p} .
\end{aligned}
$$

If $v_{k+1}$ exists, then the following relations hold:

$$
\begin{aligned}
& v_{k}^{-p^{k}}=\left(\prod_{i=0}^{r-1} \varepsilon_{i}^{p^{k} x_{i k}}\right) v_{k+1}^{-p^{k+1}}, \\
& v_{k+1}^{1-\gamma}=\left(\prod_{i=0}^{r-1} \varepsilon_{i}^{y_{i k}-y_{r k} x_{i k}}\right) v_{k+1}^{p y_{r k}} .
\end{aligned}
$$

The first step is to find $a_{i j} \in \mathbb{Z}$ which satisfy

$$
\varepsilon_{j}^{1-\gamma}=\prod_{i=0}^{r} \varepsilon_{i}^{a_{i j}} \quad(0 \leqslant j \leqslant r) .
$$

This is straightforward because

$$
\begin{aligned}
& \varepsilon_{j}^{1-\gamma}=\varepsilon_{j} \varepsilon_{j+1}^{-1} \quad(0 \leqslant j \leqslant r-1), \\
& \varepsilon_{r}^{1-\gamma}=\varepsilon_{0} \varepsilon_{1} \ldots \varepsilon_{r-1} \varepsilon_{r}^{2} .
\end{aligned}
$$

Then $A_{0}=\left(a_{i j}\right)$ is the representation matrix of $1-\gamma: V_{0} \longrightarrow V_{0}$ with respect to the basis $\left\{\varepsilon_{0}, \varepsilon_{1}, \ldots, \varepsilon_{r-1}, v_{0}\right\}$. It is easy to see that the rank of $A_{0}$ modulo $p$ is $r$ and $\operatorname{dim} \operatorname{Ker}\left(A_{0}\right.$ : $\left.\mathbb{F}_{p}^{r+1} \ni x \mapsto A_{0} x \in \mathbb{F}_{p}^{r+1}\right)=1$. Let $x_{i 0} \equiv r-i+1(\bmod p)(0 \leqslant i \leqslant r)$ with $0 \leqslant x_{i 0} \leqslant p-1$ and put ${ }^{t} x_{0}=\left(x_{00}, x_{10}, \ldots, x_{r 0}\right) \in \mathbb{Z}^{r+1}$. Then there exists ${ }^{t} y_{0}=\left(y_{00}, y_{10}, \ldots, y_{r 0}\right) \in \mathbb{Z}^{r+1}$ satisfying $A_{0} x_{0}=p y_{0}$. By the assumption $e_{1}-e_{0}=1$, we see that $\left|E_{n}^{\prime} / \mathcal{E}_{n}\right| \geqslant p$ and there exists $v_{1} \in E_{n}^{\prime}$ which satisfies (3.1) and (3.2) for $k=0$. It is straightforward to see that

$$
\varepsilon_{r-1}^{1-\gamma}=\left(\prod_{i=0}^{r-1} \varepsilon_{i}^{x_{i 0}}\right) \varepsilon_{r-1} v_{1}^{-p} .
$$


From (3.5) and (3.4), we immediately construct the representation matrix $A_{1}$ of $1-\gamma: V_{1} \longrightarrow$ $V_{1}$ with respect to the basis $\left\{\varepsilon_{0}, \varepsilon_{1}, \ldots, \varepsilon_{r-1}, v_{1}\right\}$. The first $r-1$ columns of $A_{0}$ and $A_{1}$ coincide. The last two columns vary.

When we construct $v_{k+1}$ from $v_{k}$ for $k \geqslant 1$, we need some trials. We note the following property of the representation matrix $A_{k}$ of $1-\gamma: V_{k} \longrightarrow V_{k}$ with respect to the basis $\left\{\varepsilon_{0}, \varepsilon_{1}, \ldots, \varepsilon_{r-1}, v_{k}\right\}$.

Lemma 3.1. For any $k \geqslant 1$, the rank of $A_{k}$ modulo $p$ is greater than $r-2$.

Proof. The determinant of the $(r-1) \times(r-1)$ matrix obtained from the first $r-1$ rows and $r-1$ columns of $A_{k}$ is 1 .

Namely, $\operatorname{dim} \operatorname{Ker}\left(A_{k}: \mathbb{F}_{p}^{r+1} \ni x \mapsto A_{k} x \in \mathbb{F}_{p}^{r+1}\right) \leqslant 2$ and we easily find ${ }^{t} x_{k}=$ $\left(x_{0 k}, x_{1 k}, \ldots, x_{r k}\right) \in \mathbb{Z}^{r+1}$ with $0 \leqslant x_{i k} \leqslant p-1(0 \leqslant i \leqslant r), x_{r k}=1$ and ${ }^{t} y_{k}=$ $\left(y_{0 k}, y_{1 k}, \ldots, y_{r k}\right) \in \mathbb{Z}^{r+1}$ which satisfy $A_{k} x_{k}=p y_{k}$. Starting with $v_{1}$, we try to find $v_{2}, v_{3}, \ldots, v_{n}$. If $v_{1}, \ldots, v_{k}$ exist and $v_{k+1}$ does not exist, then we have $\left(E_{n}^{\prime}: \mathcal{E}_{n}\right)=p^{k}$. Note that $A_{k+1}$ is constructed using the relations (3.3)-(3.5).

A naive method constructing $V_{k+1}$ from $V_{k}$ needs $p^{r}$ trials. A sophisticated idea of Zassenhaus in $\left[6\right.$, p. 66] reduces it to $p r$ trials but usually requires an integral basis of $K_{n}$. Our method does not need an integral basis and finds $v_{k+1}$ within $p$ trials.

\section{Examples}

We carry out practical calculations when $p=3$ and try to apply our technique to determinethe Iwasawa $\lambda$-invariant $\lambda\left(K_{\infty} / K\right)$. In the preceding paper [3], we studied $\lambda\left(K_{\infty} / K\right)$ for several imaginary quadratic fields $K=\mathbb{Q}(\sqrt{-m})$. We showed $\lambda\left(K_{\infty} / K\right)=0$ for most of these $K$. Values of $m$ for which we were not able to assert $\lambda\left(K_{\infty} / K\right)=0$ are $-2183,-4637,-6761,-7907$ and -17786 . For these $m$, we calculate the 3 -part $3^{e_{n}}$ of the ideal class number $h\left(K_{n}\right)$ of $K_{n}$.

The first step is the calculation of $e_{1}$. This is easily done because the rank of $\mathcal{E}_{1}$ is 2 , and $E_{1}^{\prime}$ is constructed straightforwardly. We verified $e_{1}-e_{0}=1$ for all above $m$. So we are able to calculate $e_{n}$ according to the technique in the previous section. We show the results in the following table, from which we see $\lambda\left(K_{\infty} / K\right)=0$ for all those $K$ using Theorem 1 in [2].

\begin{tabular}{c|cccccc}
\hline \multicolumn{1}{c}{$m$} & $e_{0}$ & $e_{1}$ & $e_{2}$ & $e_{3}$ & $e_{4}$ & $e_{5}$ \\
\hline-2183 & 1 & 2 & 3 & 4 & 4 & 4 \\
-4637 & 1 & 2 & 3 & 4 & 4 & 4 \\
-6761 & 1 & 2 & 3 & 4 & 5 & 5 \\
-7907 & 1 & 2 & 3 & 4 & 4 & 4 \\
-17786 & 2 & 3 & 4 & 5 & 6 & 6 \\
\hline
\end{tabular}

\section{Miscellaneous techniques in calculations}

We explain how we calculate special values of Siegel functions quickly and how we construct the cube root of an integer of $K_{n}$. First we consider the expression of an integer of $K_{n}$.

When $n=1$, a well-known method due to Pohst and Zassenhaus enables us to construct an integral basis of $K_{n}$ easily. When $n=2$, we used a special techniques to construct an integral basis of $K_{n}$ in [3]. It seems very hard to get an integral basis when $n \geqslant 3$. So we adopt another method. Let $\alpha$ be an integer of $K_{n}$ not contained in $K_{n-1}$. Then

$$
f_{n, \alpha}(X)=\prod_{\sigma \in \operatorname{Emb}\left(K_{n}, \mathbb{C}\right)}\left(X-\alpha^{\sigma}\right)
$$


is an irreducible polynomial in $\mathbb{Z}[X]$, where $\operatorname{Emb}\left(K_{n}, \mathbb{C}\right)$ means the set of all embeddings of $K_{n}$ into $\mathbb{C}$. Then $f_{n, \alpha}(X)$ is considered to express $2 \cdot 3^{n}$ conjugates of $\alpha$. We can specify $\alpha$ rigorously by using $f_{n, \alpha}(X)$ and an approximate value of $\alpha$ with an appropriate precision. Namely, we use a correspondence

$$
\alpha \longleftrightarrow\left\{\begin{array}{l}
\text { approximate values of } \alpha^{\sigma}, \quad \sigma \in \operatorname{Emb}\left(K_{n}, \mathbb{C}\right), \\
f_{n, \alpha}(X) .
\end{array}\right.
$$

Next we discuss how to get a cube root of $\alpha$ for an integer $\alpha$ in $K_{n}$. If one of the cube roots of $\alpha$ is contained in $K_{n}$, then only one of them, which we write $\sqrt[3]{\alpha}$, is contained in $K_{n}$ because $\zeta_{3}$ is not contained in $K_{n}$. We note the following fact.

LEMMA 5.1. Let $\alpha$ be an integer of $K_{n}$. If $f_{n, \alpha}(X)=g(X)^{3^{e}}$ with an irreducible monic polynomial $g(X) \in \mathbb{Z}[X]$ and a non-negative integer $e$, then $\alpha \in K_{n-e}$. More precisely, we have $K_{n-e}=\mathbb{Q}(\alpha)$ and $g(X)=f_{n-e, \alpha}(X)$.

Proof. If $e=0$, then the assertion is trivial. So we assume $1 \leqslant e \leqslant n$. Let $G\left(K_{n} / K\right)=\langle\gamma\rangle$. Then,

$$
\operatorname{Emb}\left(K_{n}, \mathbb{C}\right)=\left\{\gamma^{i} \mid 0 \leqslant i \leqslant 3^{n}-1\right\} \cup\left\{\gamma^{i} J \mid 0 \leqslant i \leqslant 3^{n}-1\right\},
$$

where $J$ is the complex conjugation. First, we claim that $\alpha=\alpha^{\gamma^{i}}$ for some $0<i<3^{n}$. Indeed, if $\alpha \neq \alpha^{\gamma^{i}}$ for any $0<i<3^{n}$, then we have

$$
\alpha=\alpha^{\gamma^{i} J}=\alpha^{\gamma^{j} J}
$$

for some $0 \leqslant i<j \leqslant 3^{n}-1$, which yields $\alpha=\alpha^{\gamma^{j-i}}$. This is a contradiction.

Let $i$ be the least positive integer such that $\alpha=\alpha^{\gamma^{i}}$ and put $i=3^{a} b$ with an integer $b$ prime to 3 . Since $\gamma^{3^{n}}=1$, we have

$$
\alpha=\alpha^{\gamma^{3^{a}}}
$$

which leads to $b=1$ because of the minimality of $i=3^{a} b$. Since

$$
G\left(K_{n} / K_{a}\right)=\left\langle\gamma^{3^{a}}\right\rangle
$$

we have $\alpha \in K_{a}-K_{a-1}$ and

$$
f_{n, \alpha}(X)=g(X)^{3^{e}}=h(X)^{3^{n-a}}
$$

for some monic polynomial $h(X) \in \mathbb{Z}[X]$. Since $g(X)$ is irreducible, we have $e \geqslant n-a$ and

$$
h(X)=g(X)^{3^{e+a-n}} .
$$

If $e+a>n$, then the above argument implies $\alpha \in K_{a-1}$. This contradicts the fact that $\alpha \in K_{a}-K_{a-1}$. Hence we have $e+a=n$ and complete the proof.

Lemma 5.2. Assume that $K_{n}=\mathbb{Q}(\alpha)$ with an integer $\alpha$ in $K_{n}$.

(1) If $f_{n, \alpha}\left(X^{3}\right)$ is irreducible over $\mathbb{Q}$, then $\sqrt[3]{\alpha} \notin K_{m}$ for all $m \geqslant n$.

(2) If $f_{n, \alpha}\left(X^{3}\right)=g_{1}(X) g_{2}(X)$ with an irreducible polynomial $g_{1}(X) \in \mathbb{Z}[X]$ of degree $2 \cdot 3^{n}$ and an irreducible polynomial $g_{2}(X) \in \mathbb{Z}[X]$ of degree $4 \cdot 3^{n}$, then $\sqrt[3]{\alpha} \in K_{n}$.

Proof. The proof is straightforward, noting that $\zeta_{3} \notin K_{n}$.

According to the above lemmas, we obtain $\sqrt[3]{\alpha}$ for an integer $\alpha$ of $K_{m}$ as follows. Factoring $f_{m, \alpha}(X)$, we find $n$ with $0 \leqslant n \leqslant m$ and the minimal polynomial $f_{n, \alpha}(X)$ of $\alpha$. If $f_{n, \alpha}\left(X^{3}\right)$ is irreducible, then $\sqrt[3]{\alpha} \notin K_{m}$. If $f_{n, \alpha}\left(X^{3}\right)$ has an irreducible factor $g(X)$ of degree $2 \cdot 3^{n}$, then 
$\sqrt[3]{\alpha} \in K_{m}$. Let $\sigma$ be an element of $\operatorname{Emb}\left(K_{m}, \mathbb{C}\right)$. Then $\sqrt[3]{\alpha}{ }^{\sigma}$ is one of $\rho \zeta^{i}(i=0,1,2)$, where $\rho$ is a fixed cube root of $\alpha^{\sigma}$. We specify $\rho \zeta^{i}$ so that $g\left(\rho \zeta^{i}\right)=0$. In this manner, we get the minimal polynomial of $\sqrt[3]{\alpha}$ and all conjugates of $\sqrt[3]{\alpha}$ explicitly.

Finally, we make a remark on the calculation of Siegel functions. In [3], we needed approximate values of $g\left(a_{1}, a_{2}\right)(\tau)$ with the precision of several thousand digits and calculated the infinite product straightforwardly. In this paper, we calculated the 3-part of the class number of $K_{5}$ and needed approximate values with $10^{5}$ digits. So we translated an infinite product into an infinite sum.

Lemma 5.3. Let $q_{\tau}$ and $q_{z}$ be complex numbers defined in $\S 1$. Then we have

$$
-\left(1-q_{z}\right) \prod_{n=1}^{\infty}\left(1-q_{\tau}^{n} q_{z}\right)\left(1-q_{\tau}^{n} q_{z}^{-1}\right)=q_{z}^{1 / 2} \frac{\sum_{n=0}^{\infty}(-1)^{n}\left(q_{z}^{n+1 / 2}-q_{z}^{-n-1 / 2}\right) q_{\tau}^{n(n+1) / 2}}{1+\sum_{n=1}^{\infty}(-1)^{n}\left(q_{\tau}^{n(3 n-1) / 2}+q_{\tau}^{n(3 n+1) / 2}\right)} .
$$

Proof. See [1, Proposition 6.3.14 and Corollaries 6.3.16 and 6.3.18].

REMARK 1. The convergence of the left-hand side depends on $q_{\tau}^{n}$. On the other hand, the right-hand side converges very quickly because it depends essentially on $q_{\tau}^{n^{2}}$.

REMARK 2. When $a_{1}=0, q_{z}=e^{2 \pi i a_{2}}$ is a purely imaginary number and it happens that $q_{z}^{n+1 / 2}=q_{z}^{-n-1 / 2}$ for small $n$. So we have to stop summing based on the magnitude of $\left|q_{\tau}^{n(n+1) / 2}\right|$.

REMARK 3. There is another way to use the $\sigma$-function to construct ray class fields of imaginary quadratic fields. But the $\sigma$-function needs calculations of quasi-periods which are essentially the sum of $q_{\tau}^{n}$. Though the Siegel function is similar to the $\sigma$-function, it does not need quasi-periods and hence has an advantage of fast convergence.

\section{References}

1. H. Cohen, Advanced topics in computational number theory, Graduate Texts in Mathematics 193 (Springer, Berlin, 2000).

2. T. FukUdA, 'Remarks on $\mathbb{Z}_{p}$-extensions of number fields', Proc. Japan Acad. Ser. A Math. Sci. 65 (1989) 260-262.

3. T. Fukuda and K. Komatsu, 'Non-cyclotomic $\mathbb{Z}_{p}$-extensions of imaginary quadratic fields', Exp. Math. 11 (2002) 469-475.

4. R. Gillard, 'Fonctions $L p$-adiques des corps quadratiques imaginaires et de leurs extensions abéliennes', J. reine angew. Math. 358 (1985) 76-91.

5. D. S. Kubert and S. LAng, Modular units, Grundlehren der Mathematischen Wissenschaften 244 (Springer, 1981).

6. M. E. Pонsт, 'Computing invariants of algebraic number fields', Group theory, algebra, and number theory (ed. H. G. Zimmer; de Gruyter, 1996) 53-73.

7. L. Schneps, 'On the $\mu$-invariant of $p$-adic $L$-functions attached to elliptic curves with complex multiplication', J. Number Theory 25 (1987) 20-33.

\section{T. Fukuda}

Department of Mathematics

College of Industrial Technology

Nihon University, 2-11-1 Shin-ei

Narashino, Chiba 275-8576

Japan

fukuda.takashi@nihon-u.ac.jp
K. Komatsu

Department of Mathematical Science

School of Science and Engineering

Waseda University, 3-4-1 Okubo

Shinjuku, Tokyo 169-8555

Japan

kkomatsu@waseda.jp 